\title{
Mass Spectrometry in Epigenetic Studies on Disease: Current Progress, Limitation, and Prospective
}

\section{Kangling Zhang*}

Kangling Zhang, Department of pharmacology and toxicology, University of Texas, Galvesaton, TX, USA

\section{Epigenetic Modifications and Relevance in Disease}

Though genetic mutations of genes were traditionally thought as the origin of cancer, the profoundly distorted epigenetic landscape presented in cancer cells has recently been dominating cancer research. Great progress has been made in the description of epigenetic modifications in normal and cancer tissues as exemplified by the phenomenal discoveries: Associated with cancer is DNA hypomethylation at repetitive sequences and the promoters of certain oncogenes in parallel with DNA hypermethylation at specific $\mathrm{CpG}$ islands and $\mathrm{CpG}$ island shores of promoters of cancer suppressor genes and other genes involved in the main cellular pathways including DNA repair, vitamin response, Ras signaling, cell cycle control and apoptosis [1]. On the other hand, global DNA is statistically hypomethylated in many cancer tissues as compared with normal tissues [2]. Cytosine methylation is achieved by DNA methyltransferase (DNMT) in the presence of $S$-Adenosyl methionine (SAM) and the methyl mark can be removed (demethylation) by oxidation with enzyme Tet1 in the presence of cofactors $a \mathrm{KG}$ and $\mathrm{Fe}$ (II) to form 5-hydroxylmethyl cytosine $(5 \mathrm{hmC}), 5$-fromyl-cytosine $(5 \mathrm{fC})$ and 5-carboxyl cytosine $(5 \mathrm{caC})$ followed by decarboxylation through base excision repair (BER) utilizing thymine DNA glycosylase (TDG) pathways [3]. In addition, all these cytosine derivatives could lead to either replicationdependent passive demethylation or active demethylation via excision by DNA glycosylases (TDG, SMUG1) followed by BER or even directly reciprocal conversion between $5 \mathrm{hmC}$ and C (Figure 1) [4-6]. However, how these DNA demethylation intermediate products are differentially distributed and regulated in normal and cancer cells is not clear, except emerging lines of evidence that 5-hydroxyl cytosine is absent in many cancer cells while rich in neuron stem cells [7] implicate the possibility of cancer originating from irregular epigenetic regulation in stem cell maintenance and differentiation.

Another epigenetic modification alteration in cancer cells is histone modifications. Histones composed of the nucleosome scaffold core histones ( $\mathrm{H} 2 \mathrm{~A}, \mathrm{H} 2 \mathrm{~B}, \mathrm{H} 3$, and $\mathrm{H} 4)$ and the linker histone $\mathrm{H} 1$. All histones undergo a diverse array of post-translational modifications including acetylation, methylation, phosphorylation, and ubiquitination (Figure 2). Though a converged uniform change of histone modification patterns from healthy cells to cancer cells is not known, change of modifications at specific sites was found in association with certain cancer cells. For example, global reduction of H4K16 acetylation and H4K20 trimethylation [8] accompanying a global loss of the active mark H3K4 trimethylation [9] and a gain in repressive mark H3K9/K27 methylation was found in several solid tumor cells [10,11]. The loss or gain of histone modifications is due mainly to the aberrant expression of histone modification enzymes or cofactors. The aberrant expression of these modification enzymes could result either from their own mutation or from repression by other regulatory machineries. For the local genes, DNA methylation together with histone deacetylaton and repressive histone methylation generally form a mutually reinforcing silencing loop that contributes to tumor-suppressor gene inactivation, while DNA demethylation together with histone acetylation and active histone methylation form mutually enhancing activation loci that contribute to oncogene activation in cancer cells [12].

\section{Mass Spectrometry for Global Histone Modifications and DNA Methylation}

The contribution of mass spectrometry to the epigenetics field is owing to its powerful sequencing capacity that helped identify dozens of new modification sites and types and expand the knowledge of the locations of modifications previously discovered by Edman degradation at the $\mathrm{N}$-terminus and $\mathrm{C}$-terminus to the whole protein sequence. Normally, proteins are first cut by a protease, such as trypsin, into mass spectrometry-favorable peptides and then the nature of modification at a specific site, together with the peptide sequence, is unambiguously determined by mass spectrometry. For example, a small difference in mass between acetylation and tri-methylation can be distinguished by high-accuracy mass measurement alone [13]. On the other hand, due to their relatively small size $(\sim 10-20 \mathrm{KDa})$ suitable for protein sequencing, histones can be analyzed by a high-resolution mass spectrometer to map modification sites. As an example of this, Fourier transforms ion cyclotron resonance (FT-ICR) mass spectrometry has revealed 74 modification isoforms coexisting in histone H4 [14]

Genomic DNA methylation and demethylation intermediates are normally analyzed by LC-MS after DNA is converted into nucleosides via digestion with nucleases [15] or into free bases through hydrolysis with high concentration of formic acid and at high temperature [16]. The free bases can also be analyzed by GC-MS after their functional groups $\left(-\mathrm{OH}\right.$ and $\left.-\mathrm{NH}_{2}\right)$ are chemically modified with a volatile derivative reagent such as $N$-( $t$-butyldimethylsilyl)- $N$-methyltrifluoroacetamide (MTBSTFA) with $1 \%$ tert-butyldimethylchlorosilane (TBDMCS) to facilitate GC separation and MS detection [17]

\section{Limitation of Mass Spectrometry in Epigenetic Studies}

Mass spectrometry analysis is an irreversible consumption process. Analytes are first gasified, ionized under high voltage ranged from 1 $\mathrm{KV}$ to $5 \mathrm{KV}$ depending on sampling flow rate, separated by a mass analyzer, and then detected by a detector. Though the sensitivities of modern mass spectrometers have improved dramatically in recent years, MS still lacks the capability of identifying epigenetic modifications in an individual gene, because MS does not have the same sequence-reading and concentration-amplification functions as the chromatin immunoprecipitation (ChIP) experiment that uses primers complementary to the target region along with a polymerasechain-reaction (PCR) to enable selective and repeated amplification. Therefore, the latter is currently a common and often indispensable

${ }^{*}$ Corresponding author: Kangling Zhang, Department of pharmacology and toxicology, University of Texas, Galvesaton, TX, USA, Tel: 409 772-9650; E-mail: kazhang@UTMB.EDU

Received May 13, 2013; Accepted May 13, 2013; Published May 15, 2013

Citation: Zhang K (2013) Mass Spectrometry in Epigenetic Studies on Disease: Current Progress, Limitation, and Prospective. Mol Biol 2: e109. doi:10.4172/21689547.1000e109

Copyright: (c) 2013 Zhang K. This is an open-access article distributed under the terms of the Creative Commons Attribution License, which permits unrestricted use, distribution, and reproduction in any medium, provided the original author and source are credited. 


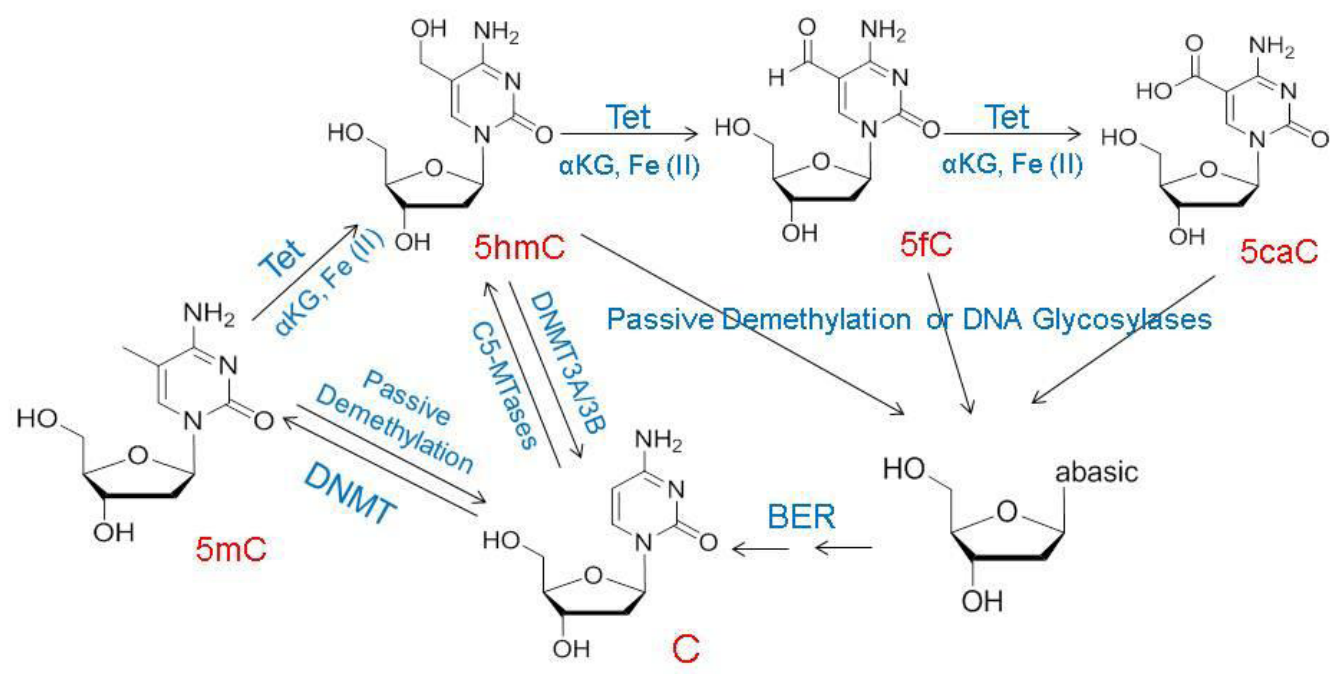

Figure 1: DNA methylation and demethylation.

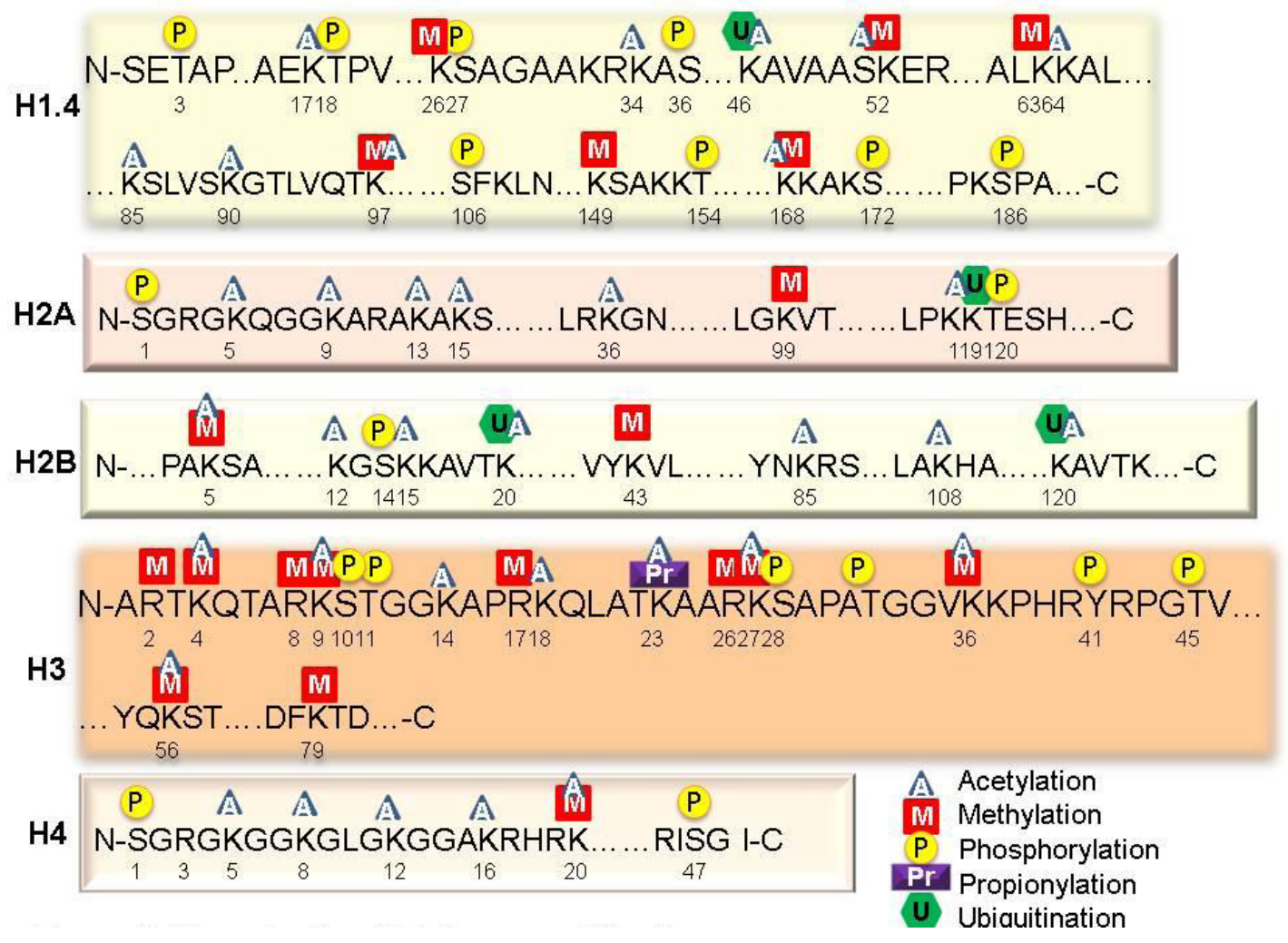

Figure 2: Complexity of histone modification

technique for assaying epigenetic modifications at gene loci. In contrast, MS is definitely advantageous over the antibody-based techniques (ca. Western-blot) for assaying global epigenetic modifications, thanks to its relatively high sensitivity and particularly, unmatchable selectivity. However, the lower ionization efficiency and subsequent poorer sensitivity of detecting whole proteins than peptides by FTICR limits its applications in epigenetic studies in which sample size of clinical samples is restricted. Therefore, MS-based determination of histone modifications for clinical samples is made primarily by the quantification of modifications in tryptic peptides. However, several technical drawbacks of the peptide method remain to be addressed. For instance, there is a challenge to quantify a peptide, such as $\mathrm{H} 3$ K27xxxK36 peptide (Figure 2), that contains multiple methylation sites because of multilayer analytical complexity: each methylation site can be mono-, di-, and tri-methylated; all the methylated peptide isoforms including isobaric isoforms are eluted from the HPLC column at almost the same retention time; and there is a substantial difference in signal response to concentration among mono-, di-, and tri-methylation. Secondly, there isn't an integrated mass spectrometry method to assess histone modifications and DNA methylation simultaneously 
because different procedures and treatments are used for preparation of histones and DNA samples. With one single analytical method, the cross-talk between DNA methylation and histone modifications could be directly assessed and the analytical bias in two otherwise separated analytical methods would be eliminated.

\section{Prospective}

Mass spectrometry with a scan mode of selective ion monitoring (SIM) or selective and multiple reaction monitoring (SRM and MRM) may be the method of choice for quantification of epigenetic modifications with unmatched sensitivity and selectivity. Our laboratory has innovatively applied the LC-MS/MS-MRM method that is widely utilized for the quantification of small molecules particularly in pharmaceutical industries to the quantification of histone modifications [18]. We are currently improving this method to absolutely quantify histone modifications in SILAC-histones (SILAC: Stable isotope labelling by amino acids in cell culture) using synthetic stable-isotope labelled modified peptides. Further, SILAC-histones will be used as modified histone internal standards to quantify histone modifications in samples of interest. It is expected that such an absolute quantification approach will be able to reveal accurate histone modification status in complex biological system including solving the above-mentioned $\mathrm{H} 3$ K27/K36 methylation complexity. We are also developing LC-MS/MSMRM method to simultaneously quantify histone modifications and DNA methylation in isolated chromatins so that the cross-talk between DNA methylation and histone modifications can be directly evaluated with one single analytical method. With these methods developed, we will be able to perform robust epigenetic network studies in biological systems including neuron stem cell differentiation, monocyte-tomacrophage differentiation, environmental stressed diseases, and cancer etiology.

\section{References}

1. Portela A, Esteller M (2010) Epigenetic modifications and human disease. Nat Biotechnol 28: 1057-1068.

2. Ehrlich M (2009) DNA hypomethylation in cancer cells. Epigenomics 1: 239259

3. He YF, Li BZ, Li Z, Liu P, Wang Y, et al. (2011) Tet-mediated formation of 5 -carboxylcytosine and its excision by TDG in mammalian DNA. Science 333: 1303-1307.

4. Auclair G, Weber M (2012) Mechanisms of DNA methylation and demethylation in mammals. Biochimie 94: 2202-2211.
5. Liutkeviciute Z, Lukinavicius G, Masevicius V, Daujotyte D, Klimasauskas S (2009) Cytosine-5-methyltransferases add aldehydes to DNA. Nat Chem Biol 5: $400-402$.

6. Chen CC, Wang KY, Shen CK (2012) The mammalian de novo DNA methyltransferases DNMT3A and DNMT3B are also DNA 5-hydroxymethylcytosine dehydroxymethylases. J Biol Chem 287: 3311633121.

7. Kriaucionis S, Heintz N (2009) The nuclear DNA base 5-hydroxymethylcytosine is present in Purkinje neurons and the brain. Science 324: 929-930.

8. Fraga MF, Ballestar E, Villar-Garea A, Boix-Chornet M, Espada J, et al. (2005) Loss of acetylation at Lys 16 and trimethylation at Lys20 of histone $\mathrm{H} 4$ is a common hallmark of human cancer. Nat Genet 37: 391-400.

9. Hamamoto R, Furukawa Y, Morita M, limura Y, Silva FP, et al. (2004) SMYD3 encodes a histone methyltransferase involved in the proliferation of cancer cells. Nat Cell Biol 6: 731-740.

10. Kondo Y, Shen L, Suzuki S, Kurokawa T, Masuko K, et al. (2007) Alterations of DNA methylation and histone modifications contribute to gene silencing in hepatocellular carcinomas. Hepatol Res 37: 974-983.

11. Viré E, Brenner C, Deplus R, Blanchon L, Fraga M, et al. (2006) The Polycomb group protein $\mathrm{EZH} 2$ directly controls DNA methylation. Nature 439: 871-874.

12. Sharma S, Kelly TK, Jones PA (2010) Epigenetics in cancer. Carcinogenesis 31: $27-36$.

13. Zhang K, Siino JS, Jones PR, Yau PM, Bradbury EM (2004) A mass spectrometric "Western blot" to evaluate the correlations between histone methylation and histone acetylation. Proteomics 4: 3765-3775.

14. Phanstiel D, Brumbaugh J, Berggren WT, Conard K, Feng X, et al. (2008) Mass spectrometry identifies and quantifies 74 unique histone $\mathrm{H} 4$ isoforms in differentiating human embryonic stem cells. Proc Natl Acad Sci USA 105: 4093-4098.

15. Le T, Kim KP, Fan G, Faull KF (2011) A sensitive mass spectrometry method for simultaneous quantification of DNA methylation and hydroxymethylation levels in biological samples. Anal Biochem 412: 203-209.

16. Zhang L, Zhang L, Zhou K, Ye X, Zhang J, et al. (2012) Simultaneous determination of global DNA methylation and hydroxymethylation levels by hydrophilic interaction liquid chromatography-tandem mass spectrometry. J Biomol Screen 17: 877-884.

17. Herring JL, Rogstad DK, Sowers LC (2009) Enzymatic methylation of DNA in cultured human cells studied by stable isotope incorporation and mass spectrometry. Chem Res Toxicol 22: 1060-1068.

18. Darwanto A, Curtis MP, Schrag M, Kirsch W, Liu P, et al. (2010) A modified "cross-talk" between histone H2B Lys-120 ubiquitination and H3 Lys-79 methylation. J Biol Chem 285: 21868-21876. 